comprised observations on the sun, planets, and daylight stars, as well as night observing. Star observations, taken usually before dusk and after dawn, provided corrections to reduce sun and planet observations to a night basis. These results were then solved for corrections to the equinox, the equator, and the orbital elements.

The catalogue of 1589 stars comprises 180 clock stars, 2 I azimuth stars, and stars between $-30^{\circ}$ and $+90^{\circ}$ declination selected from the Backlund-Hough list, given in the supplement to the Connaissance des Temps of r9I4. Eichelberger's positions were used for the clock stars, whereas the positions of the azimuth stars were determined from Ottawa observations, using Eichelberger's proper motions. Positions were finally reduced to 1925.0 without proper motion.

The declinations were computed from nadir readings, using $45^{\circ} 23^{\prime} 39^{\prime \prime}$. o as a provisional latitude of the meridian circle.

Corrections were applied for horizontal flexure, variation of latitude, as given in the International Latitude Publications, and refraction based on Bessel's Tabulae Regiomontanae. A solution of pole stars at upper and lower culmination gave a corrected value of latitude $45^{\circ} 23^{\prime} \quad 38$ '.89. Screens were used to reduce star magnitudes, and a reversing prism was used with the eyepiece throughout the work.

Comparisons with the $\mathrm{GC}$ and $\mathrm{FK}_{3}$ are given for 3-hour intervals, as well as the observed corrections to $\mathrm{GC}$ and $\mathrm{FK}_{3}$ for the epoch of observation. Equinox and equator corrections were deduced but were not applied to the observations.

Results for this same list of stars taken, 19351950, with eye- and object-end reversed are now ready for publication. The right ascension will be based on the same clock stars as the two previous publications but on the $\mathrm{FK}_{3}$ system.

Since 1950 observations are being taken on special stars needed for photographic zenith tube work. The star list was made up from stars used at Richmond, Florida, at Ottawa, and the list proposed for PZT work at Herstmonceux. These are reduced to mean place to the end of I952.

Commencing in 1954 it is proposed to observe all $\mathrm{FK}_{3}$ stars to declination $-27^{\circ} 3 \mathrm{O}^{\prime}$ and the supplemental list of $\mathrm{FK}_{3}$ stars making a total of approximately 3000 stars.

Dominion Observatory, Ottarea, Canada, 1953 September.

\title{
ON THE ACCURACY ATTAINABLE BY STATISTICAL REDUCTION TO ABSOLUTE PROPER MOTION
}

\section{By A. N. VYSSOTSKY}

\begin{abstract}
The accuracy of reduction of relative proper motions to absolute values depends on both observational and cosmical errors. In the case of long-focus telescopes it is important to limit the choice of reference stars to faint stars of early spectral types. For shorter instruments the observational errors are much larger than the cosmical errors and the final accuracy may be considerably increased by using a greater number of stars and a longer time interval. Tables I to $\mathrm{V}$ contain the errors computed for various instruments and various circumstances of observation.
\end{abstract}

I. Two methods for the reduction of the relative photographic proper motion of a star are available: a) simultaneous observation of one or more stars with known absolute motion in the same field; b) the use of the mean motion of the reference stars as determined elsewhere statistically.

The first method has very limited application. Stars with well determined absolute proper motions are not numerous and in addition they are usually much too bright and may consequently introduce photographic magnitude errors. The more numerous absolute motions of the fainter stars in the Yale zone catalogues (photo- graphic A.G.C.) have not as yet been reduced to a uniform absolute system and their accuracy is not sufficiently high.

Consequently in many problems, such as the derivation of proper motions of Cepheids, cluster-type variables, long-period variables, etc., it is necessary to use statistical reductions. We shall now examine the errors which are introduced in this process.

2. When the proper motion of a star is referred to the mean motion of a group of stars, two types of error should be considered: a) the error of observation, i.e. bisecting, film, and measuring machine errors, as well as distortions 
of the field, collimation, etc.; and b) the so-called cosmical error, in other words, the error in the group motion of the reference stars traceable to their peculiar motions and their limited numbers. Ideally, the errors of observation can be reduced as much as desired: a) by the use of long-focus refractors; b) by increasing the number of pairs of plates; c) by increasing the time interval between the first and the last epochs. The cosmical error, however, cannot be reduced by any of these means, since its value is determined by the size of the peculiar motions of the reference stars and the number of such stars available. Thus, in order to reduce the cosmical error, one should choose as many reference stars as possible and stars whose peculiar motions are expected to be small. For instance, the choice may be limited to stars of spectral classes A and F, or to stars of faint apparent magnitude, say, fourteenth photographic.

3. The final step in the reduction of the relative proper motion to an absolute value is the computation of the parallactic motion of the reference stars. The secular parallaxes of stars of various spectral classes down to photovisual magnitude II.o have been published by the McCormick Observatory, ${ }^{1}$ and the mean parallaxes for fainter stars without respect to spectral type down to photographic magnitude I 4.0 by Binnendijk. ${ }^{2}$ The formal probable errors of these parallaxes are sufficiently small so that they will not appreciably increase the total probable error. It should be noted, however, that Binnendijk's values have been obtained by assuming dispersions for faint stars in high latitudes which have not yet been verified. In fact practically the only radial velocity material available is that of Edmondson for stars of the tenth magnitude, ${ }^{3}$ which leads us to suspect that Binnendijk's assumed dispersions may be as much as 50 per cent too small. It is true that any error in his assumption will be partly offset by a compensating error in the value assumed for the solar velocity relative to these stars, but still there is the possibility of a 25 per cent error in the secular parallaxes of faint stars in high latitudes.

4. Tables I to III contain the relevant probable errors which contribute to the formation of the total error. It will be seen from Table II that the limitation of reference stars in low latitudes to spectral classes $\mathrm{A}$ and $\mathrm{F}$ considerably reduces the cosmical errors in this zone. In Table III the cosmical errors are computed from Binnendijk's values of mean parallaxes ${ }^{2}$ and Oort's values of
TABLE I. ERRORS OF OBSERVATION FOR ONE PAIR OF PLATES (UNIT $=0$ ".OOOI)

\begin{tabular}{lllllc} 
Focal & & \multicolumn{5}{c}{ Interval in Yea } \\
Length & \multicolumn{1}{c}{ Scale } & 20 & 40 & 60 & Notes \\
$32.6 \mathrm{ft}$. & $2 \mathrm{I}^{\prime \prime} / \mathrm{mm}$ & 30 & 15 & 10 & I \\
I I.3 & 60 & 65 & 32 & 22 & 2 \\
I I .7 & 57 & 45 & 23 & I 5 & 3 \\
I I.7 & 57 & 70 & 35 & 23 & 4
\end{tabular}

Notes: I. McCormick 26-inch refractor; $P u b . M c C$. Obs. 7, 2, 1937. 2. Normal Astrograph; Pub. Poulkovo Obs. 55, I8, 1940. 3. Lick Astrograph, stars; A.J. 58, I26, 1953. 4. Lick Astrograph, nebulae (l.c.).

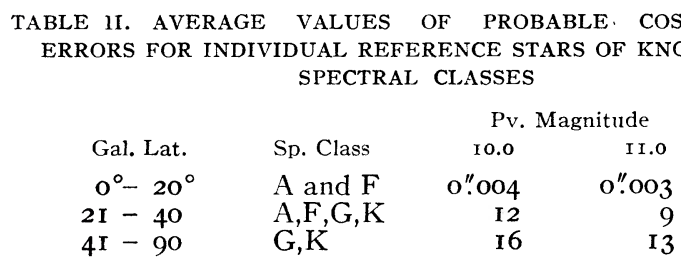

Source: $P u b . M c C$. Obs. 1o, Tables 8, VII, 9. III, 9. IX; 9, Table I (p. I38); I2, Table I2. I; A.J. 54, 35, I 948.

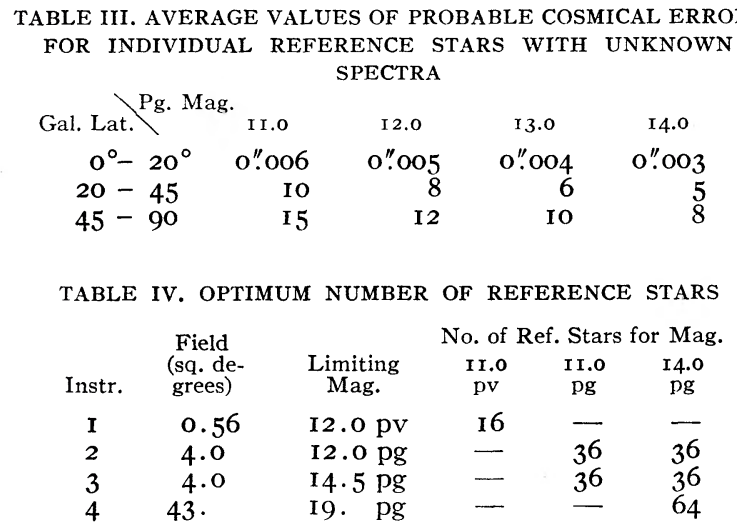

Instrument: I. McCormick 26-inch; 2. Normal Astrograph (Catalogue plates); 3. Normal Astrograph (Chart plates); 4. Lick Astrograph.

peculiar velocities. ${ }^{4}$ Table IV indicates the practical number of reference stars when size of field, limiting magnitude, etc. are taken into account. Thus: a) the fainter the stars, the smaller the cosmical errors; b) secular parallaxes for stars fainter than 14.5 mag. are extrapolations of low accuracy ; c) the work of measuring and computing increases nearly in proportion to the number of reference stars while the probable cosmical error of the mean motion cannot possibly decrease more rapidly than the square root of the number of these stars.

Table $\mathrm{V}$ gives the resulting total probable errors as derived from the preceding Tables for various circumstances of observation. Thus, 


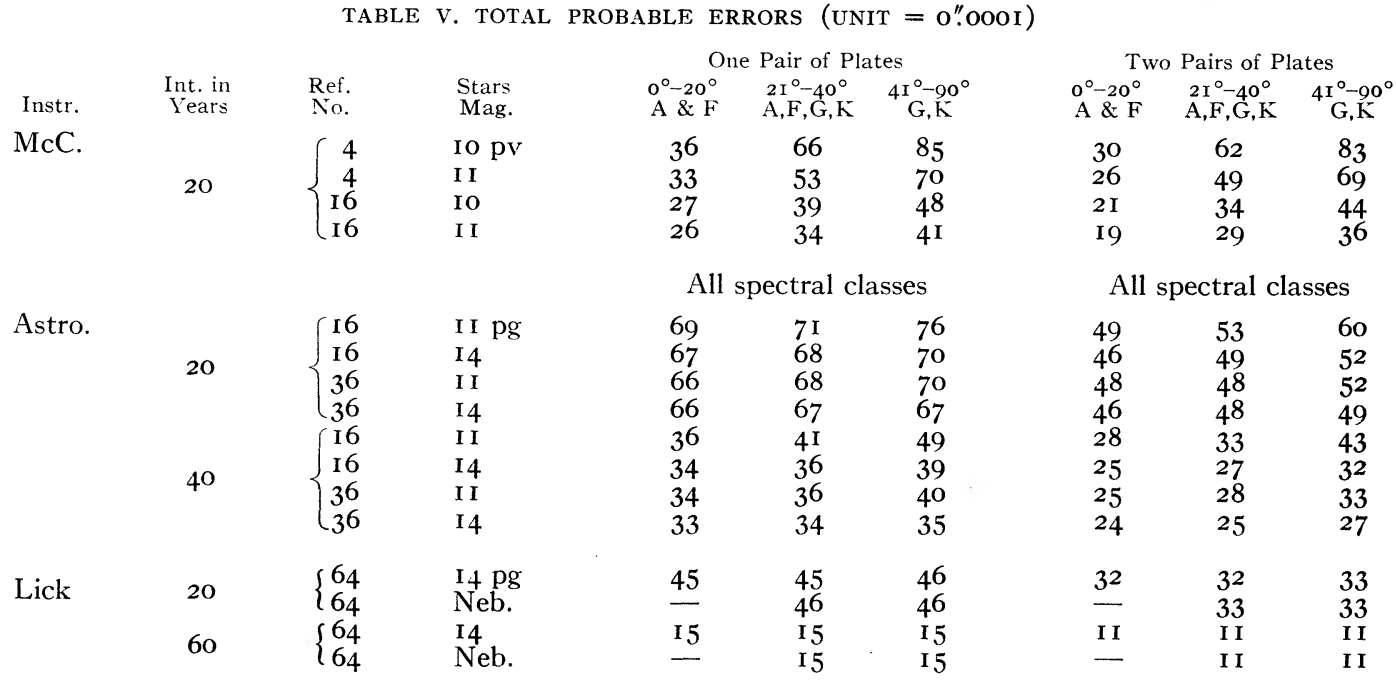

consider a program star which appears in an Astrographic Catalogue of 40 years ago and for which 36 reference stars of eleventh magnitude are available in low latitudes. We have:

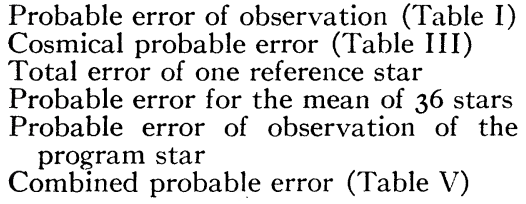

To this combined probable error must be added vectorially the probable error of the parallactic motion as determined at Leiden or McCormick. This will vary, or course, with the region of the sky and will be negligible except in high latitudes, as noted in Section 3.

It is clear from Table $\mathrm{V}$ that a program of determining proper motions in high latitudes at McCormick Observatory will be successful only when the expected annual motions of the program stars are greater than o".oro, since we cannot always be sure of having sixteen reference stars in our limited field. An increase in the time interval or in the number of pairs of plates would not improve matters in this case.

The situation is reversed however when smaller instruments are used, such as an Astrographic telescope or the Lick Astrograph. Here, by increasing the time interval and the number of pairs of plates, we can improve the accuracy very considerably, and the chief consideration is the amount of work involved.

5. Finally it may be well to say a word about the treatment of reference stars with large motions. It is, of course, a statistical outrage to discard a reference star completely simply because it has a large motion. Relative motions obtained in such a manner cannot possibly be reduced to absolute motion by statistical tables.

On the other hand, Oort has devised a procedure for dealing with these stars which in effect reduces the cosmical errors involved. ${ }^{4}$ Thus, he temporarily discards stars with motions exceeding a given limit and subsequently adjusts his data by allowing for the motions of all the discarded stars. This is a delicate operation and not to be recommended except in most experienced hands; it should be accompanied by complete details of the exclusions and adjustments, so that subsequent investigators may be able to make use of the material in any way which may later appear desirable.

\section{REFERENCES}

I. Pub. McCormick Obs. 10, p. 36, 1948 .

2. B. A. N. го, p. 9.

3. A. J. 54, 35, 1948.

4. B. A. N. 8, 75, 1936. 